\title{
Corrigendum
}

\section{An anticancer C-Kit kinase inhibitor is reengineered to make it more active and less cardiotoxic}

Ariel Fernández, Angela Sanguino, Zhenghong Peng, Eylem Ozturk, Jianping Chen, Alejandro Crespo, Sarah Wulf, Aleksander Shavrin, Chaoping Qin, Jianpeng Ma, Jonathan Trent, Yvonne Lin, Hee-Dong Han, Lingegowda S. Mangala, James A. Bankson, Juri Gelovani, Allen Samarel, William Bornmann, Anil K. Sood, and Gabriel Lopez-Berestein

Original citation: J Clin Invest. 2007;117(12):4044-4054. doi:10.1172/JCI32373.

Citation for this corrigendum: J Clin Invest. 2013;123(11):4980. doi:10.1172/JCI73378.

During the preparation of this manuscript, information regarding an NIH grant was omitted from the Acknowledgments. The correct Acknowledgments section is below.

The research of A. Fernández was supported by NIH grant R01 GM072614 and by a grant from the Gulf Coast Center for Computational Cancer Research. J. Ma acknowledges support from the Welch Foundation. G. Lopez-Berestein acknowledges support from CA. A.K. Sood acknowledges support from the University of Texas MD Anderson Cancer Center SPORE in ovarian cancer (P50CA83639) and a Program Project Development Grant from the Ovarian Cancer Research Fund Inc. The GIST882 cells were a gift from Jonathan Fletcher, Dana-Farber Cancer Institute-Harvard Medical School. The experimental findings reported in Figure 5 validating the theoretical results in the paper were obtained in compliance with the aims and collaborative agreements with Eli Lilly and Co. specified in the NIH/NIGMS grant R01GM072614 (A. Fernandez, principal investigator).

The authors regret the error.

\section{Erratum}

Depleting tumor-specific Tregs at a single site eradicates disseminated tumors

Aurélien Marabelle, Holbrook Kohrt, Idit Sagiv-Barfi, Bahareh Ajami, Robert C. Axtell, Gang Zhou, Ranjani Rajapaksa, Michael R. Green, James Torchia, Joshua Brody, Richard Luong, Michael D. Rosenblum, Lawrence Steinman, Hyam I. Levitsky, Victor Tse, and Ronald Levy

Original citation: J Clin Invest. 2013;123(6):2447-2463. doi:10.1172/JCI64859.

Citation for this erratum: J Clin Invest. 2013;123(11):4980.doi:10.1172/JCI73340.

During the editing process, the survival curves in Figure 4G were mislabeled. The correct figure is below.

The JCI regrets the error.

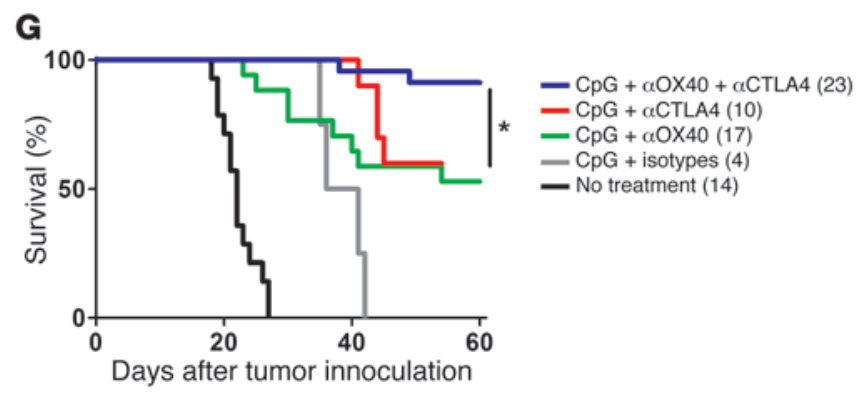

